\title{
Harmonizing Formula Prescription Patterns for Chronic Kidney Disease: A Population-Based Cross- Sectional Study
}

\section{Hung-Lung Lin}

Kaohsiung Medical University Hospital, Kaohsiung Medical University

Ming-Yen Lin

Kaohsiung Medical University Hospital https://orcid.org/0000-0002-8194-4833

Cheng-Hsun Tasi

Kaohsiung Medical University Hospital, Kaohsiung Medical University

\section{Yi-Hsiu Wang}

Kaohsiung Medical University Hospital, Kaohsiung Medical University

\section{Chung-Jen Chen}

Kaohsiung Medical University Hospital, Kaohsiung Medical University

\section{Shang-Jyh Hwang}

Kaohsiung Medical University

Yi-Wen Chiu ( $\nabla$ chiuyiwen@gmail.com )

https://orcid.org/0000-0001-6840-5440

\section{Research}

Keywords: Chinese herbal medicine, chronic kidney disease, indications, complementary and alternative medicine

Posted Date: April 8th, 2020

DOl: https://doi.org/10.21203/rs.3.rs-20650/v1

License: (c) (1) This work is licensed under a Creative Commons Attribution 4.0 International License. Read Full License 


\section{Abstract}

\section{Background}

Harmonizing formulas have been demonstrated to be associated with reduced risk of end-stage renal disease in patients with chronic kidney disease (CKD). However, the target population and indications of harmonizing formulas in CKD remain unknown.

\section{Methods}

We conducted a population-based cross-sectional study to explore factors associated with harmonizing formulas prescription. Patients who had been prescribed harmonizing formulas after CKD diagnosis were defined as the using harmonizing formulas group. Disease diagnoses for harmonizing formula prescriptions and patient characteristics related to the prescriptions were collected.

\section{Results}

In total, 24,971 patients were enrolled in this analysis, and 5,237 (21\%) patients were prescribed harmonizing formulas after CKD diagnosis. The three most frequent systematic diseases and related health problems for which harmonizing formula prescriptions were issued in CKD were symptoms, signs, and ill-defined conditions (24.5\%), diseases of the digestive system, $(20.67 \%)$, and diseases of the musculoskeletal system (12.9\%). Higher likelihoods of harmonizing formula prescriptions were associated with young age (adjusted odds ratio: $0.98,95 \%$ confidence interval: $0.97-0.98$ ), female sex $(1.79,1.68-1.91)$, no diabetes $(1.20,1.06-1.35)$, no hypertension $(1.38,1.26-1.50)$, no cerebrovascular

disease $(1.34,1.14-1.56)$, less disease severity $(0.86,0.83-0.89)$, using nonsteroidal anti-inflammatory drugs (NSAIDs) $(1.65,1.54-1.78)$, and using analgesic drugs other than NSAIDs $(1.46,1.35-1.59)$.

\section{Conclusions}

Harmonizing formulas are commonly used for treating symptoms of the digestive and musculoskeletal systems in CKD cases. Further research on harmonizing formula effectiveness with regard to particular characteristics of CKD patients is warranted.

\section{Background}

Chronic kidney disease (CKD), defined as substantial renal damage persisting for 3 months, is prevalent and affects $8-16 \%$ of adults globally (1). CKD can be regarded as an irreversible condition. After a patient develops $C K D$, renal function might progressively deteriorate over several years until reaching to end-stage renal disease (ESRD) (2). Although guidelines have recommended that CKD should be appropriately managed to slow its progression $(3,4)$, maintaining stable renal function and relieving the symptoms and signs caused by accumulation of uremic toxins remain challenging. Chinese herbal medicines (CHMs), through syndrome differentiation and treatment selection, present a means to improve CKD care and may stabilize renal function. 
Several experimental animal studies have concluded that certain herbs (e.g., Radix Salvia miltiorrhizae, Rhubarb) in CHM have potential anti-inflammatory and antifibrotic effects and could be an agent in renal fibrosis therapy $(5,6)$. However, these findings are less applicable in clinical practice because illnesses in humans are often more complex than those in mice. For example, CKD in humans may have different etiologies and be accompanied by noncommunicable diseases. Therefore, observational human studies of $\mathrm{CHM}$ treatment effects provide an opportunity to understand current practices in CHM therapy and could help in exploring new therapeutic formulas for use in CKD. Studies and our previous data have demonstrated the potential beneficial effects of CHM on CKD outcomes (7-10), highlighting that further understanding of the target population in CKD for treatment with specific formulas is warranted.

Harmonizing formulas are commonly used in treating disorders caused by contrasting illnesses including shaoyang (half interior and half exterior) syndromes, liver and spleen disharmonies, and intestine and stomach disharmonies (11). The effectiveness of harmonizing formulas has been widely recognized in reducing depressive syndromes $(12,13)$ and improving survival in patients with stroke and cancer $(14)$. However, applications for patients with CKD remain relatively less understood. Our study explored disease categories for which prescribing harmonizing formulas could be appropriate and independent factors associated with harmonizing formula prescriptions in patients with CKD.

\section{Methods}

\section{Data Source and Subjects}

The Taiwan government launched the National Health Insurance (NHI) program in 1995. It covers $99.6 \%$ of Taiwan's population and has service contracts with $93 \%$ of health care institutes. NHI reimburses medications (Western medicine and CHM), ambulatory, emergency, dental, and inpatient care after health care institutes complete medical services and uploads claims data. The claims data are further managed by the Taiwan National Health Research Institutes for inclusion in the National Health Insurance Research Database (NHIRD) and are available for academic research.

We conducted a population-based cross-sectional study by using the Longitudinal Health Insurance Database 2000 (LHID2000), a subset of the NHIRD. The LHID2000 contains the data of 1 million randomly sampled patients who were $\mathrm{NHI}$ beneficiaries in 2000 . The randomly sampled patients exhibit similar distributions in age, sex, birth year, and average insured payroll-related amount with the general population.

\section{CKD}

The detailed methods of this study were in our previous study (10). In brief, we identified patients who received new diagnoses of CKD between 2000 and 2005 by using the frequency of appearance of specific International Classification of Diseases, Ninth Revision, Clinical Modification (ICD-9-CM) codes within one year (15). Although laboratory data were lacking in these databases, we can reasonably 
assume that most of these patients had stage 3-5 CKD [estimated glomerular filtration rate (eGFR) < $60 \mathrm{ml} / \mathrm{min} / 1.73 \mathrm{~m}^{2}$ ] on the basis of regional hospital data using the same algorithm. We excluded patients who were aged $<18$ or $\geq 85$ years $(n=2,673)$, had cancer $(n=1,680)$ or underwent dialysis before receiving a CKD diagnosis $(n=28)$, or had received any CHM prescription within 1 year before diagnosis $(n=11,104)$. Therefore, the cohort was suitable to assess for determining factors associated with CHM prescription.

\section{Harmonizing Formulas}

All CHM prescriptions from CKD diagnosis to the start of dialysis or the end of 2008 were collected. We distinguished these $\mathrm{CHM}$ prescriptions into harmonizing formulas and other formulas according to approaches suggested in textbooks, with minor modifications $(11,16)$. To detect potential indicators for using harmonizing formulas, disease categories for prescribing harmonizing and other formulas were compared on the basis of a patient's first ICD-9-CM diagnosis code of the prescription and classified into various disease system and health problem groups. These systematic diseases and related health problems can be divided into infections and parasitic diseases (ICD-9-CM: 001-139); neoplasms (140239); endocrine, nutritional, and metabolic diseases, and immunity disorders (240-279); diseases of the blood and blood-forming organs (280-289); mental disorders (290-319); diseases of the nervous system and sensory organs (320-389); diseases of the circulatory system (390-459); diseases of the respiratory system (460-519); diseases of the digestive system (520-579); diseases of the genitourinary system (580-629); complications of pregnancy, childbirth, and the puerperium (630-679); diseases of the skin and subcutaneous tissue (680-709); diseases of the musculoskeletal system (710-739); congenital anomalies (740-759); certain conditions originating in the perinatal period (760-779); symptoms, signs, and ill-defined conditions (780-799); injury and poisoning (800-999); and supplementary classification and others (V01-V82, E800-E999).

\section{Assessment of Associated Factors}

We collected information on patient characteristics, namely age, sex (male or female), insurance amount (fixed premium or dependent, <New Taiwan dollar (NT\$)20,000, NT\$20,000-39,999, and $\geq$ NT\$39,999 [ US\$1,333]), region (north, center, south, and east), urbanization of residence (urban or rural), main comorbidities (acute coronary syndrome, diabetes, hypertension, hyperlipidemia, chronic obstructive pulmonary disease (COPD), and cerebrovascular disease), Charlson comorbidity index score, and primary Western medicines used (diabetic drugs, antihypertensive drugs, nonsteroidal anti-inflammatory drugs (NSAIDs), analgesic drugs other than NSAIDs, and antilipid drugs) during the observation period.

\section{2-1. Statistical Analysis}

Continuous and categorical data were expressed as mean \pm standard deviation or median (interquartile range) and percentage, respectively. Significant differences in patient characteristics between the harmonizing formula use and nonuse groups were evaluated using an independent $t$ test for continuous variables and $\chi^{2}$ test for categorical variables. We used the proportion of disease categories to determine the differences in disease treatment between using harmonizing formulas and other formulas. In 
addition, a multivariable logistic regression with forced entry of all collected factors was performed to identify the independent factors of harmonizing formula prescription. Results of the logistic regression were represented as odds ratios (ORs) and 95\% confidence intervals (Cls). All statistical operations were performed in SAS (version 9.4, SAS Institute, Cary, NC, USA). A $P$ value $<0.05$ was considered significant.

\section{Results}

\section{Patient Characteristics by Use of Harmonizing Formulas}

We included 24,971 patients who received new diagnoses of CKD, and $21 \%$ of these patients were prescribed harmonizing formulas. Compared with patients in the harmonizing formulas nonuse group, patients in the group using harmonizing formulas were significantly more likely to be young, female, and living in central Taiwan and urban areas. They were significantly more likely to have high insurance amounts, fewer comorbidities (acute coronary syndrome, diabetes, hypertension, hyperlipidemia, COPD, or cerebrovascular disease), less disease severity, and less use of diabetic and antihypertensive drugs, but more use of NSAIDs, analgesic drugs other than NSAIDs, and antilipid drugs.

\section{Disease Categories for Prescribing Harmonizing Formulas}

The disease categories for prescribing harmonizing formulas and the other types of Chinese herbal formulas in patients with CKD were compared and are listed in Table 2. The three most frequent disease categories for prescribing harmonizing formula in CKD were symptoms, signs, and ill-defined conditions (24.5\%); diseases of the digestive system (20.67\%); and diseases of the musculoskeletal system (12.9\%). Similar frequencies of disease categories were observed for other formula prescriptions in patients with CKD. Notably, an increased proportion of patients with diseases of the digestive system $(6.39 \%$ difference) and a reduced proportion of those with diseases of the respiratory system ( $-5.5 \%$ difference) received harmonizing formula prescriptions compared with other formula prescriptions in this analysis. 
Table 1

Characteristics of study cohort by harmonizing formula use

\begin{tabular}{|c|c|c|c|}
\hline \multirow[b]{2}{*}{ Characteristic } & \multicolumn{2}{|c|}{ Harmonizing formulas } & \multirow[t]{2}{*}{$P$ value } \\
\hline & Nonuse & Use & \\
\hline Patient no. & 19734 & 5237 & \\
\hline Age, y & $58.3 \pm 16.4$ & $51.3 \pm 15.6$ & $<0.001$ \\
\hline Female (\%) & 37.9 & 50.2 & $<0.001$ \\
\hline Insurance amount, NT\$ (\%) & & & $<0.001$ \\
\hline Fixed premium or dependent & 20.5 & 18.8 & \\
\hline$<20,000$ & 58.1 & 56.4 & \\
\hline $20,000-39,999$ & 13.7 & 16.4 & \\
\hline$\geqq 39,999$ & 7.7 & 8.5 & \\
\hline Region (\%) & & & $<0.001$ \\
\hline North & 46.6 & 43.9 & \\
\hline Center & 21.1 & 23.6 & \\
\hline South & 28.5 & 29.7 & \\
\hline East & 3.7 & 2.8 & \\
\hline Urbanization (\%) & & & $<0.001$ \\
\hline Urban & 72.9 & 76.6 & \\
\hline \multicolumn{4}{|l|}{ Comorbidities (\%) } \\
\hline Acute coronary syndrome & 12.5 & 7.7 & $<0.001$ \\
\hline Diabetes & 29.9 & 19.4 & $<0.001$ \\
\hline Hypertension & 40.7 & 26.6 & $<0.001$ \\
\hline Hyperlipidemia & 13.5 & 11.1 & $<0.001$ \\
\hline
\end{tabular}

Abbreviations: COPD, chronic obstructive pulmonary disease; IQR, interquartile range; NSAIDs, nonsteroidal anti-inflammatory drugs; NTS, New Taiwan Dollar.

The differences in characteristics among groups were compared using $\chi^{2}$ tests for categorical variables and independent $t$ tests for continuous variables. A $P$ value of $<0.05$ was considered statistically significant.

NT\$30 equals approximately US\$1. 


\begin{tabular}{|c|c|c|c|}
\hline \multirow[b]{2}{*}{ Characteristic } & \multicolumn{2}{|c|}{ Harmonizing formulas } & \multirow[t]{2}{*}{$P$ value } \\
\hline & Nonuse & Use & \\
\hline COPD & 9.2 & 5.4 & $<0.001$ \\
\hline Cerebrovascular disease & 11.5 & 4.3 & $<0.001$ \\
\hline \multicolumn{4}{|l|}{ Charlson score } \\
\hline Mean \pm SD & $1.49 \pm 1.87$ & $0.82 \pm 1.28$ & $<0.001$ \\
\hline Median (IQR) & $1(0-2)$ & $0(0-1)$ & $<0.001$ \\
\hline \multicolumn{4}{|l|}{ Confounding drugs, $\%$} \\
\hline Diabetic drugs & 29.7 & 24.6 & $<0.001$ \\
\hline Antihypertensive drugs & 55.6 & 51.1 & $<0.001$ \\
\hline NSAIDS & 29.4 & 40.6 & $<0.001$ \\
\hline Analgesic drugs other than NSAIDs & 42.9 & 45.8 & $<0.001$ \\
\hline Anti-lipid drugs & 20.9 & 21.1 & $<0.001$ \\
\hline \multicolumn{4}{|c|}{$\begin{array}{l}\text { Abbreviations: COPD, chronic obstructive pulmonary disease; IQR, interquartile range; NSAIDs, } \\
\text { nonsteroidal anti-inflammatory drugs; NTS, New Taiwan Dollar. }\end{array}$} \\
\hline \multicolumn{4}{|c|}{$\begin{array}{l}\text { The differences in characteristics among groups were compared using } \chi^{2} \text { tests for categorical } \\
\text { variables and independent } t \text { tests for continuous variables. A } P \text { value of }<0.05 \text { was considered } \\
\text { statistically significant. }\end{array}$} \\
\hline NT\$30 equals approximately US\$1. & & & \\
\hline
\end{tabular}


Table 2

Systematic diseases and related health problems for harmonizing formula prescription claims

\begin{tabular}{|c|c|c|c|c|}
\hline Disease category (ICD-9-CM) & $\begin{array}{l}\text { Harmonizing } \\
\text { formulas (a) }\end{array}$ & $\begin{array}{l}\text { Other } \\
\text { formulas } \\
\text { (b) }\end{array}$ & $\begin{array}{l}\text { Difference } \\
{[(a)-(b)]}\end{array}$ & $\begin{array}{l}\text { Ratio } \\
{[(a) /(b)]}\end{array}$ \\
\hline Infection and parasitic diseases $(001-139)$ & 0.87 & 0.52 & 0.35 & 1.67 \\
\hline Neoplasms (140-239) & 0.78 & 0.68 & 0.10 & 1.15 \\
\hline $\begin{array}{l}\text { Endocrine, nutritional, and metabolic diseases } \\
\text { and immunity disorders }(240-279)\end{array}$ & 3.52 & 5.02 & -1.5 & 0.7 \\
\hline $\begin{array}{l}\text { Diseases of the blood and blood-forming } \\
\text { organs }(280-289)\end{array}$ & 0.32 & 0.37 & -0.05 & 0.86 \\
\hline Mental disorders (290-319) & 1.76 & 0.9 & 0.86 & 1.96 \\
\hline $\begin{array}{l}\text { Diseases of the nervous system and sense } \\
\text { organs }(320-389)\end{array}$ & 3.37 & 3.71 & -0.34 & 0.91 \\
\hline Diseases of the circulatory system $(390-459)$ & 2.49 & 4.29 & -1.80 & 0.58 \\
\hline Diseases of the respiratory system $(460-519)$ & 6.54 & 12.09 & -5.55 & 0.54 \\
\hline Diseases of the digestive system $(520-579)$ & 20.67 & 14.28 & 6.39 & 1.45 \\
\hline $\begin{array}{l}\text { Diseases of the genitourinary system (580- } \\
629 \text { ) }\end{array}$ & 12.38 & 11.93 & 0.45 & 1.04 \\
\hline $\begin{array}{l}\text { Complications of pregnancy, childbirth, and } \\
\text { the puerperium }(630-679)\end{array}$ & 0.17 & 0.18 & -0.01 & 0.94 \\
\hline $\begin{array}{l}\text { Diseases of the skin and subcutaneous tissue } \\
(680-709)\end{array}$ & 2.10 & 2.90 & -0.80 & 0.72 \\
\hline $\begin{array}{l}\text { Diseases of the musculoskeletal system } \\
(710-739)\end{array}$ & 12.9 & 12.51 & 0.39 & 1.03 \\
\hline Congenital anomalies $(740-759)$ & 0.37 & 0.28 & 0.09 & 1.32 \\
\hline $\begin{array}{l}\text { Certain conditions originating in the perinatal } \\
\text { period }(760-779)\end{array}$ & 0.01 & 0.00 & 0.01 & - \\
\hline $\begin{array}{l}\text { Symptoms, signs, and ill-defined conditions } \\
(780-799)\end{array}$ & 24.50 & 24.54 & -0.04 & 1.00 \\
\hline
\end{tabular}

Abbreviations: ICD-9-CM, International Classification of Diseases, Ninth Revision, Clinical Modification; aOR, adjusted odds ratio; $\mathrm{Cl}$, confidence interval.

Number of Chinese medicine visits is 24,226 for harmonizing formulas and 622,790 for other formulas.

Proportion of disease categories by harmonizing and other formulas are displayed. 


\begin{tabular}{|lllll|}
\hline Disease category (ICD-9-CM) & $\begin{array}{l}\text { Harmonizing } \\
\text { formulas }(\mathbf{a})\end{array}$ & $\begin{array}{l}\text { Other } \\
\text { formulas } \\
(\mathbf{b})\end{array}$ & $\begin{array}{l}\text { Difference } \\
{[(\mathbf{a})-(\mathbf{b})]}\end{array}$ & $\begin{array}{l}\text { Ratio } \\
{[(\mathbf{a}) /(\mathrm{b})]}\end{array}$ \\
\hline Injury and poisoning (800-999) & 5.48 & 3.51 & 1.97 & 1.56 \\
\hline $\begin{array}{l}\text { Supplementary classification and others } \\
\text { (V01-V82, E800-E999) }\end{array}$ & 1.78 & 2.27 & -0.49 & 0.78 \\
\hline $\begin{array}{l}\text { Abbreviations: ICD-9-CM, International Classification of Diseases, Ninth Revision, Clinical } \\
\text { Modification; aOR, adjusted odds ratio; Cl, confidence interval. }\end{array}$ & \\
\hline $\begin{array}{l}\text { Number of Chinese medicine visits is 24,226 for harmonizing formulas and 622,790 for other } \\
\text { formulas. }\end{array}$ & \\
\hline
\end{tabular}

\section{Prescription Frequency of Constituent Herbs in Harmonizing Formulas}

As Fig. 1 presents, the three most frequently prescribed herbs for harmonizing formulas during the observed period were Jia Wei Xiao Yao San, Shao Yao Gan Cao Tang, and Xiao Chai Hu Tang. The most frequent disease categories for Jia Wei Xiao Yao San prescriptions were symptoms, signs, and ill-defined conditions ( $n=2,143$ times); genitourinary disease $(n=1,767$ times); and diseases of the digestive system ( $n=1,157$ times). The most frequent disease category for Shao Yao Gan Cao Tang prescriptions was diseases of the musculoskeletal system ( $n=1,876$ times). Furthermore, the most frequent disease category for Xiao Chai Hu Tang prescription was diseases of the digestive system ( $n=1,056$ times).

\section{Factors Associated With Harmonizing Formulas Prescription}

Table 3 presents the associations of baseline patient characteristics with harmonizing formula prescriptions. Patients who were young (adjusted OR: 0.98, 95\% Cl: 0.97-0.98); female (1.79, 1.68-1.91); had high insurance amounts (range of adjusted OR: 1.14-1.31); lived in central Taiwan (1.26, 1.15-1.37), southern Taiwan $(1.20,1.11-1.30)$, or urban areas $(1.16,1.07-1.26)$; did not have diabetes $(1.20,1.06-$ $1.35)$, hypertension $(1.38,1.26-1.50)$, or cerebrovascular disease $(1.34,1.14-1.56)$; had lower disease severity $(0.86,0.83-0.89)$; used NSAIDs $(1.65,1.54-1.78)$; and used analgesic drugs other than NSAIDs $(1.46,1.35-1.59)$ were more likely to have harmonizing formula prescriptions. 
Table 3

Factors Associated with Harmonizing Formula Prescription in Patients with Chronic Kidney Disease

\section{Adjusted odds ratio $(95 \% \mathrm{Cl})$}

$P$ value

Age, $y$

$0.98(0.97-0.98)$

$<0.001$

Female (male as reference)

$1.79(1.68-1.91)$

$<0.001$

Insurance amount, NT\$ (\%)

Fixed premium or dependent

1.00 [Ref.]

$<20,000$

$1.14(1.05-1.24)$

0.004

$20,000-39,999$

$1.25(1.12-1.40)$

$<0.001$

$\geqq 39,999$

$1.31(1.15-1.50)$

$<0.001$

Region (\%)

North

1.00 [Ref.]

Center

$1.26(1.15-1.37)$

$<0.001$

South

$1.20(1.11-1.30)$

$<0.001$

East

$0.88(0.73-1.08)$

0.22

Urbanization (\%)

Rural

1.00 [Ref.]

Urban

$1.16(1.07-1.26)$

$<0.001$

Comorbidities (Yes as reference)

No acute coronary syndrome

$1.01(0.89-1.14)$

0.88

No diabetes

$1.20(1.06-1.35)$

0.005

No hypertension

$1.38(1.26-1.50)$

$<0.001$

No hyperlipidemia

$1.02(0.92-1.14)$

0.67

No COPD

$0.87(0.75-1.01)$

0.06

No cerebrovascular disease

$1.34(1.14-1.56)$

$<0.001$

Abbreviations: COPD, chronic obstructive pulmonary disease; NSAIDs, nonsteroidal anti-inflammatory drugs; NT\$, New Taiwan dollar; $\mathrm{Cl}$, confidence interval.

Multiple logistic regression adjusted by all listed variables through the forced entry approach was used.

NT\$30 equals approximately US\$1. 
Charlson score, point

$0.86(0.83-0.89)$

$<0.001$

Confounding drugs (None as reference)

$\begin{array}{lcc}\text { Diabetic drugs } & 1.07(0.95-1.19) & 0.27 \\ \text { Antihypertensive drugs } & 1.07(0.98-1.17) & 0.10 \\ \text { NSAIDs } & 1.65(1.54-1.78) & <0.001 \\ \text { Analgesic drugs other than NSAIDs } & 1.46(1.35-1.59) & <0.001 \\ \text { Anti-lipid drugs } & 1.05(0.95-1.15) & 0.35\end{array}$

Abbreviations: COPD, chronic obstructive pulmonary disease; NSAIDs, nonsteroidal anti-inflammatory drugs; NT\$, New Taiwan dollar; Cl, confidence interval.

Multiple logistic regression adjusted by all listed variables through the forced entry approach was used.

NT\$30 equals approximately US\$1.

\section{Discussion}

The current study demonstrated that one-fifth of patients with CKD have been prescribed harmonizing formulas, which were associated with risk reduction of ESRD in our previous study (10). Symptoms, signs, and ill-defined conditions; diseases of the digestive system; and diseases of the musculoskeletal system were the three most frequent disease classifications for prescribing harmonizing formulas. Patients with CKD who were young, female, had high premiums, lived in central or southern Taiwan or urban areas, did not have comorbidities (acute coronary syndrome, diabetes, hypertension, or cerebrovascular disease), had lower disease severity, and used NSAIDs and analgesic drugs other than NSAIDs were more likely to have harmonizing formulas prescriptions.

Nearly half of incident CKD patients used conventional CHM. Harmonizing formulas accounted for $46.1 \%$ of prescriptions. Although the efficacy of harmonizing formulas in reducing depression and improving survival in patients with liver cancers and systemic lupus erythematosus have been reported in studies $(14,17)$, few studies have paid attention to prescription patterns and outcomes in treating patients with CKD $(18,19)$. Combined with the results of Yang et al. (2014), Chen et al. (2018), and our previous findings, the current study indicated that one harmonizing formula, Jia Wei Xiao Yao San, is the main prescription for patients with late CKD. This formula potentially improves patient renal function after short- or long-term observation $(10,19)$. Jia Wei Xiao Yao San is traditionally used to relieve stagnation in liver qi, reduce depression symptoms, and improve spleen qi deficiency. Although the mechanisms of delaying ESRD are complex and worthy of further study, Jia Wei Xiao Yao San may affect renal clinical outcome by improving depressive symptoms, which are a novel predictor of accelerated eGFR decrease, 
dialysis therapy initiation, death, or hospitalization $(10,20)$. We are unsure of the effect harmonizing formulas have in relieving uremic symptoms or slowing renal progression.

We attempted to use the ICD-9-CM to as a reference for the indications of harmonizing formula prescription in CKD patients. CHM has its own historical and systematic philosophy of symptom differentiation (Bian Zheng) to assess, explicate, diagnose, and treat patient symptoms. Numerous symptoms in CKD patients not requiring dialysis are either unrecognized or suboptimally managed by clinical care workers $(21,22)$. assessed 283 patients with stage 1-5 CKD and reported that tiredness (81\%; 95\% Cl: 76.0-85.6), sleep disturbance (70\%; 64.3-75.3), and pain in bones or joints $(69 \%$; 63.474.6) were the most common symptoms regardless of CKD stage. Loss of appetite, nausea, vomiting, fatigue, and edema are common symptoms in late-stage CKD, which are similar to "spleen deficiency syndrome" in CHM and tend to involve the digestive system. In addition, local pain, weakness in the loin and knee, and calf cramps are frequently encountered CKD symptoms, which are similar to "liver-kidney insufficiency syndromes" in CHM and tend to involve the musculoskeletal system. Shao Yao Gan Cao Tang, a frequently prescribed harmonizing formula in CKD, is used to relieve muscle pain or skeletal muscle tremors in Japan and China (23). However, additional beneficial effects of Shao Yao Gan Cao Tang on renal health remain unknown and warrant further investigation.

Female sex, low prevalence of comorbidity, and high use of analgesic drugs were associated with higher prescription frequency for harmonizing formulas in CKD. Although the causal relationships are difficult to establish in this study, a possible explanation is that prevalence of pain for females is high, and they are more likely to be aware of pain and receive relevant treatments $(24,25)$. NSAIDs are commonly used for pain control in clinical practice, but caution should be exercised when they are applied in CKD because they can induce more severe renal injuries (26). How harmonizing formulas interact with NSAIDs for pain control in patients with CKD remains unclear. Thus, more research is required on this combination therapy to study its efficacy in pain control in CKD and preventing further renal injury.

Our study has some advantages. First, assessments of Chinese herbal formulas in the study were drawn from a nationwide health insurance database with highly comprehensive records of CHM prescriptions. Second, CHM in this study was prescribed by quality assurance physicians who were educated in the same system and accredited by Taiwan's government; this strengthens the reliability of symptomatic differentiation and accuracy of disease diagnostic coding. Third, Taiwan's NHI is one of the few national insurance programs that reimburses both Western medicine and $\mathrm{CHM}$, providing an opportunity to explore the foundational philosophies of these two different modes for treating certain diseases. However, some limitations must be declared. First, the lack of laboratory and patient-reported data in the claims database prevents us from exploring the possible mechanisms of harmonizing formulas on health outcomes such as emotion, pain, and renal function. In addition, using the ICD-9-CM diagnosis system to identify disease classification of CHM prescriptions may not have accurately reflected the indications of $\mathrm{CHM}$ formulas. However, we believe that using the main categories of diseases partially represented the CHM diagnostic system. Finally, our results were from the NHI program having high accessibility, which may limit their generalizability. 


\section{Conclusions}

In conclusion, this study determined that harmonizing formulas are commonly used in treating CKD for symptoms, signs, and ill-defined conditions; genitourinary diseases; and digestive system diseases. Patients who were young, female, had fewer comorbidities, and used analgesic drugs were more likely to be prescribed harmonizing formulas, which suggests that more research on the efficacy of Western medicine and $\mathrm{CHM}$ in patients with CKD and these characteristics is required.

\section{List Of Abbreviations}

CHMs, Chinese herbal medicines; Cls, confidence intervals; CKD, chronic kidney disease; COPD, chronic obstructive pulmonary disease; eGFR, estimated glomerular filtration rate; ESRD, end-stage renal disease; ICD-9-CM, International Classification of Diseases, Ninth Revision, Clinical Modification; LHID2000, Longitudinal Health Insurance Database 2000; NHIRD, National Health Insurance Research Database; $\mathrm{NIH}$, National Health Insurance; NSAIDs, nonsteroidal anti-inflammatory drugs; NT\$, New Taiwan dollar; ORs, odds ratios.

\section{Declarations}

Ethics approval and consent to participate

This research was approved by the Institutional Review Board of Kaohsiung Medical University Hospital (KMUHIRB-EXEMPT(I)-20150063). All research procedures followed the guidelines of the Declaration of Helsinki.

\section{Consent for publication}

The Institutional Review Board of Kaohsiung Medical University Hospital exempted from the requirement of informed consent because the personal privacy information within the analyzed data have been completely de-identified and allowed for improvement of public interest.

Availability of data and materials

All data generated or analysed during this study are included in this published article.

Conflicts of interest

None of the authors have any conflicts of interest to declare.

\section{Funding}

This study was supported by Kaohsiung Medical University Hospital, Kaohsiung Medical University (grant numbers: KMUH104-4M55, KMUH 105-5R17, KMUH 106-6R21, KMUH 103-3R10, and KMUH 104-4R11). 
The funders had no role in the design or conduct of the study; collection, management, analysis, or interpretation of the data; or preparation, review, or approval of the manuscript.

\section{Authors' contributions}

Conceptualization, HLL, MYL, and YWC; methodology, MYL, CJC, and SJH; writing-original draft preparation, HLL, CHT and YHW; writing-review and editing, MYL, and YWC; supervision, CJC and SJH. All authors have read and agreed to the published version of the manuscript.

\section{Acknowledgments}

We would like to thank the Statistical Analysis Laboratory of the Department of Internal Medicine of Kaohsiung Medical University Hospital for providing access to the NHIRD (registration number 99324), and appreciate the efforts of Miss Teng-Hui Huang in creating the figure, and Wallace Academic Editing in editing this manuscript. Some of the results of this study were presented in abstract form at the 2016 Annual Meeting of the Taiwan Society of Nephrology.

\section{References}

1. Jha V, Garcia-Garcia G, Iseki K, Li Z, Naicker S, Plattner B, et al. Chronic kidney disease: global dimension and perspectives. The Lancet. 2013;382(9888):260-72.

2. Zhong J, Yang H-C, Fogo AB. A perspective on chronic kidney disease progression. American Journal of Physiology-Renal Physiology. 2017;312(3):F375-F84.

3. Levin A, Stevens PE, Bilous RW, Coresh J, De Francisco AL, De Jong PE, et al. Kidney Disease: Improving Global Outcomes (KDIGO) CKD Work Group. KDIGO 2012 clinical practice guideline for the evaluation and management of chronic kidney disease. Kidney International Supplements. 2013;3(1):1-150.

4. Levey AS, Coresh J, Bolton K, Culleton B, Harvey KS, Ikizler TA, et al. K/DOQI clinical practice guidelines for chronic kidney disease: evaluation, classification, and stratification. American Journal of Kidney Diseases. 2002;39(2 SUPPL. 1).

5. Zhao T, Sun S, Zhang H, Huang X, Yan M, Dong X, et al. Therapeutic Effects of Tangshen Formula on Diabetic Nephropathy in Rats. PloS one. 2016;11(1):e0147693.

6. Lu Z, Zeng Y, Lu F, Liu X, Zou C. Rhubarb Enema Attenuates Renal Tubulointerstitial Fibrosis in 5/ 6 Nephrectomized Rats by Alleviating Indoxyl Sulfate Overload. PloS one. 2015;10(12):e0144726.

7. Hsieh C-F, Chang H-C, Huang S-L, Chen C-L, Chen W-T, Yang C-C. Prescribed Renoprotective Chinese Herbal Medicines Were Associated with a Lower Risk of All-Cause and Disease-Specific Mortality among Patients with Chronic Kidney Disease: A Population-Based Follow-Up Study in Taiwan. Evidence-Based Complementary and Alternative Medicine. 2017;2017.

8. Hsieh CF, Huang SL, Chen CL, Chen WT, Chang HC, Yang CC. Non-aristolochic acid prescribed Chinese herbal medicines and the risk of mortality in patients with chronic kidney disease: results 
from a population-based follow-up study. BMJ open. 2014;4(2):e004033.

9. Hsu P-C, Tsai Y-T, Lai J-N, Wu C-T, Lin S-K, Huang C-Y. Integrating traditional Chinese medicine healthcare into diabetes care by reducing the risk of developing kidney failure among type 2 diabetic patients: a population-based case control study. J Ethnopharmacol. 2014;156:358-64.

10. Lin M-Y, Chiu Y-W, Chang J-S, Lin H-L, Lee CT-C, Chiu G-F, et al. Association of prescribed Chinese herbal medicine use with risk of end-stage renal disease in patients with chronic kidney disease. Kidney international. 2015;88(6):1365-73.

11. Chen JK, Chen TT. Chinese Herbal Formulas and Applications. City of Industry, CA, Art of Medicine Press. 2009.

12. Zeng LF, Cao Y, Wang L, Dai YK, Hu L, Wang Q, et al. Role of Medicinal Plants for Liver-Qi Regulation Adjuvant Therapy in Post-stroke Depression: A Systematic Review of Literature. Phytother Res. 2017;31(1):40-52.

13. Yeung W-F, Chung K-F, Ng K-Y, Yu Y-M, Zhang S-P, Ng BF-L, et al. Prescription of Chinese herbal medicine in pattern-based traditional Chinese medicine treatment for depression: a systematic review. Evidence-Based Complementary and Alternative Medicine. 2015;2015.

14. Liao YH, Lin CC, Lai HC, Chiang JH, Lin JG, Li TC. Adjunctive traditional Chinese medicine therapy improves survival of liver cancer patients. Liver International. 2015;35(12):2595-602.

15. Collins AJ, Chen SC, Gilbertson DT, Foley RN. CKD surveillance using administrative data: impact on the health care system. American journal of kidney diseases: the official journal of the National Kidney Foundation. 2009;53(3 Suppl 3):27-36.

16. Cao M [Wang Ang and his Variorum of medical recipes (Yi fang ji jie)]. Zhonghua yi shi za zhi (Beijing, China: 1980). 2000;30(3):179 - 81.

17. Ma Y-C, Lin C-C, Li C-I, Chiang J-H, Li T-C, Lin J-G, editors. Traditional Chinese medicine therapy improves the survival of systemic lupus erythematosus patients. Seminars in arthritis and rheumatism; 2016: Elsevier.

18. Chen W, Chen $\mathrm{H}-\mathrm{Y}$, Yang $\mathrm{Y}-\mathrm{H}$, Yang S-H, Yang C-W, Wu Y-H, et al. An investigation of the prescription patterns of chinese herbal products for chronic glomerulonephritis patients: a hospital-based crosssectional study. Evidence-Based Complementary and Alternative Medicine. 2018;2018.

19. Yang T-H, Chen H, Yang S-H, Lin Y-H, Fang J-T, Hung C-C, et al. Utilization pattern for traditional chinese medicine among late stage chronic kidney disease patients: a hospital-based cross-sectional study. Journal of Chinese Medicine. 2014;25(1):41-58.

20. Shirazian S, Grant CD, Aina O, Mattana J, Khorassani F, Ricardo AC. Depression in chronic kidney disease and end-stage renal disease: similarities and differences in diagnosis, epidemiology, and management. Kidney international reports. 2017;2(1):94-107.

21. Brown SA, Tyrer FC, Clarke AL, Lloyd-Davies LH, Stein AG, Tarrant C, et al. Symptom burden in patients with chronic kidney disease not requiring renal replacement therapy. Clinical Kidney Journal. 2017;10(6):788-96. 
22. van der Veer SN, Aresi G, Gair R. Incorporating patient-reported symptom assessments into routine care for people with chronic kidney disease. Oxford University Press; 2017.

23. Wu T-H, Chen L-C, Yang L-L. Hypouricemic effect and regulatory effects on autonomic function of Shao-Yao Gan-Cao Tang, a Chinese herbal prescription, in asymptomatic hyperuricemic vegetarians. Rheumatol Int. 2007;28(1):27-31.

24. Johannes CB, Le TK, Zhou X, Johnston JA, Dworkin RH. The prevalence of chronic pain in United States adults: results of an Internet-based survey. The Journal of Pain. 2010;11(11):1230-9.

25. Fillingim RB. Sex, gender, and pain: women and men really are different. Current review of pain. 2000;4(1):24-30.

26. Luciano R, Perazella MA. NSAIDs: acute kidney injury (acute renal failure). UpToDate, Basow DS, editor, UpToDate, Waltham. 2015.

\section{Figures}


Huang Lian

E Jiao Tang

Chai Hu Jia Long Gu

Mu Li Tang

Gan Cao

Xie Xin Tang

Sheng Jiang

Xie Xin Tang

Xiao Chai Hu Tang

Qu Ngin Sam

Chai Hu Gui Zhi

Gan Jiang Tang

Huang Lian

Tang

Chair Shiann Tang

Xiao Yao San

Chai Hu Gui Zhi Tang

Si Ni Sang

Chai Hu

Shu Gan Tang

Xiao Chai $\mathrm{Hu}$

Tang

Shao Yao

Gan Cao Tang

Jia Wei

Xiao Yao San
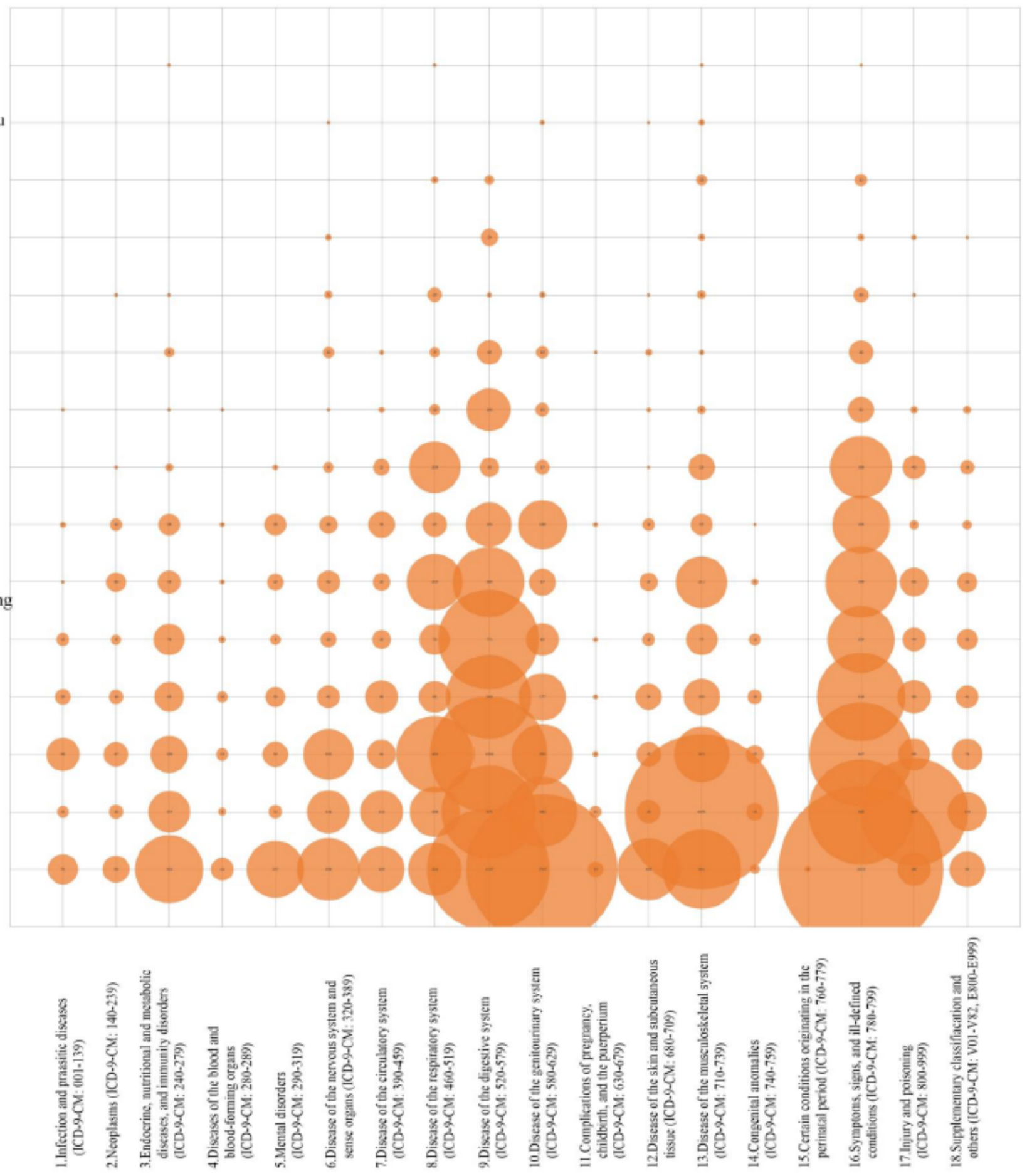

Disease name and ICD-9-CM codes

\section{Figure 1}

Prescription frequency of constituent herbs of harmonizing formulas categorized by systematic disease and related health problems in patients with chronic kidney disease. 